\title{
Fazit und Ausblick
}

Das in diesem Essentials Band dargestellte Vorgehen verfolgt drei Ziele: Erstens soll es als eine schrittweise Anleitung zum Aufbau eines Steuerungssystems für die Kommunikation in den sozialen Medien dienen. Zweitens sollen zwar die vielen verfügbaren Kennzahlen und deren einzelne Berechnungen und Deutungen verständlich vorgestellt werden, aber gleichzeitig soll durch ihre Zuordnung zu den einzelnen Phasen der Customer Journey deutlich werden, wie die verwirrende Komplexität zielorientiert reduziert werden kann. Drittens soll damit ein Weg aufgezeigt werden, wie eine erfolgsorientierte Kommunikationssteuerung auch mit verhältnismäßig einfachen Mitteln, d. h. ohne externe Analyse-Software, möglich ist, sofern sie gut vorbereitet und kontinuierlich betreut wird.

Eine hohe Datenintegration und die technische Fähigkeit den einzelnen Nutzer entlang seines individuellen Entscheidungsprozesses (Single Customer View) beobachten zu können, bieten Vorteile für die Aussteuerung von Marketingmaßnahmen durch ein personalisiertes Targeting, das auf den aktuellen Entscheidungsschritt der Nutzers abgestimmt ist, und potentiell für die Attribution der erzielten Umsätze zu den einzelnen Maßnahmen und Touchpoints. Sie sind aber keine notwendige Voraussetzung für eine erfolgsorientierte Steuerung der Unternehmenskommunikation. Mit den durch die sozialen Plattformen zur Verfügung gestellten Kennzahlen ist eine Steuerung und Optimierung der Touchpoints entlang der gesamten Customer Journey möglich, sofern das Unternehmen bereit ist, über die sehr prominent nach vorne gestellten Kennzahlen hinweg zu schauen und aufbauend auf einem Customer Journey Mapping ein Kennzahlensystem passend zu seinen operativen Marketingzielen und Möglichkeiten aufzubauen. Die Erarbeitung eines solchen Steuerungssystems ist zwar aufwendig und bedarf auch kontinuierlicher Pflege, bietet aber viel Potential für die Optimierung der unternehmenseigenen Kommunikationsaktivitäten. 